\title{
Target mass number dependence of subthreshold antiproton production in proton-, deuteron- and alpha-particle-induced reactions
}

\author{
H Müller ${ }^{1}$ and V I Komarov ${ }^{2}$ \\ ${ }^{1}$ Institut für Kern- und Hadronenphysik, Forschungszentrum Rossendorf, \\ Postfach 510119, D-01314 Dresden, Germany \\ 2 Joint Institute for Nuclear Research, LNP, 141980 Dubna, Russia \\ E-mail: H.Mueller@fz-rossendorf.de
}

\begin{abstract}
Data from KEK on subthreshold $\overline{\mathrm{p}}$ as well as on $\pi^{ \pm}$and $\mathrm{K}^{ \pm}$production in proton-, deuteron- and $\alpha$-induced reactions at energies between 2.0 and $12.0 \mathrm{~A} \mathrm{GeV}$ for $\mathrm{C}, \mathrm{Cu}$ and $\mathrm{Pb}$ targets are described within a unified approach. We use a model which considers a nuclear reaction as an incoherent sum over collisions of varying numbers of projectile and target nucleons. It samples complete events and thus allows for the simultaneous consideration of all final particles including the decay products of the nuclear residues. The enormous enhancement of the $\overline{\mathrm{p}}$ cross section, as well as the moderate increase of meson production in deuteron and $\alpha$ induced compared to proton-induced reactions, is well reproduced for all target nuclei. In our approach, the observed enhancement near the production threshold is mainly due to the contributions from the interactions of few-nucleon clusters by simultaneously considering fragmentation processes of the nuclear residues. The ability of the model to reproduce the target mass dependence may be considered as a further proof of the validity of the cluster concept.
\end{abstract}

Submitted to: J. Phys. G: Nucl. Phys.

\section{Introduction}

In two recent papers $[1,2]$ we discussed data [3] on $\overline{\mathrm{p}}, \pi^{ \pm}$and $\mathrm{K}^{ \pm}$production in the reactions $\mathrm{pC}, \mathrm{dC}$ and $\alpha \mathrm{C}$ at energies between 3.5 and $12.0 \mathrm{~A} \mathrm{GeV}$ within the Rossendorf collision (ROC) model. Special emphasis was on subthreshold production of antiprotons the enormous enhancement of which in $\mathrm{dC}$ and $\alpha \mathrm{C}$ compared to $\mathrm{pC}$ reactions could be well reproduced. Here, we consider the target mass number dependence of this effect by confronting the KEK data [3] on hadron production in $\mathrm{pCu}, \mathrm{dCu}, \alpha \mathrm{Cu}$ and $\mathrm{dPb}$ reactions with $\mathrm{ROC}$-model calculations and make predictions for the reactions $\mathrm{pPb}$ and $\alpha \mathrm{Pb}$ not measured so far. 


\section{The model}

The ROC model considers a nuclear reaction within the spectator-participant picture as an incoherent sum over collisions of varying numbers of projectile and target nucleons. Partial cross sections for these interactions are derived from a probabilistic interpretation of the Glauber theory [4] in close analogy to the cooperative model [5-12]. They depend strongly on the mass numbers via the wave functions of the nuclei. For light nuclei they drop more rapidly with increasing number of participants than in case of heavier nuclei.

Particle production is proposed to proceed via intermediate states called fireballs (FBs). This assumption yields a natural explanation of particle correlations observed at higher energies (see the discussion in [13]). Two parameters, temperature and radius, define number and relative kinetic energy of the final hadrons the FBs decay into. Resonances among the final hadrons decay later on into stable particles observed in the experiment. The nuclear residue is assumed to become excited during the reaction due to the distortion of the nuclear structure by the separation of the participants from the spectators and due to the passage of the reaction products through the spectator system. In this way, final-state interactions are taken into account without making special assumptions concerning re-absorption, re-scattering, self-energies, potentials etc for each particle type separately. Obviously, a comparison of model calculations with data for heavier nuclei should be much more sensitive to the treatment of such secondary effects.

As thoroughly discussed in [1], the consideration of the fragmentation of the nuclear residues is decisive for reproducing subthreshold cross sections. The model describes fragmentation in analogy to the decay of the excited FBs also by a temperature and a radius parameter. Obviously, the main ingredients of the model (partial cross sections, FB excitation, final-state interactions and fragmentation) may influence the total mass number dependence of hadron production cross sections quite differently. Thus, the consideration of the mass number dependence is an important check to verify the model assumptions.

The mathematical formulation of the above picture is given in [2]. In the following we employ the same parameter set as used in $[1,2]$ for the $\mathrm{C}$ target also for the heavier target nuclei except the maximum temperature of the nuclear residues (see section 3).

\section{Comparison with experimental data}

As discussed in $[1,15]$, one of the outstanding features of the ROC model is the unified treatment of particle production and fragmentation processes. The interplay of these two energy consuming processes is of special importance at subthreshold energies where the available energy is decisive for particle production cross sections. Therefore, we start our consideration of mass number dependence of (subthreshold) particle production by fixing the parameters of the nuclear residues from independent data $[14,16-19]$ for multifragmentation in proton-nucleus interactions. For simplicity, the radius parameter $R_{R}=1.7 \mathrm{fm}$ and the value of $\bar{a}=0.5$ (equation (17) in [2]) defining the dependence of the temperature parameter $\Theta_{R}$ from the number of participants $a$

$$
\Theta_{R}(a, A)=\Theta_{R}^{\max }\left[1-\exp \left(-a / \bar{a} A^{1 / 3}\right)\right]
$$




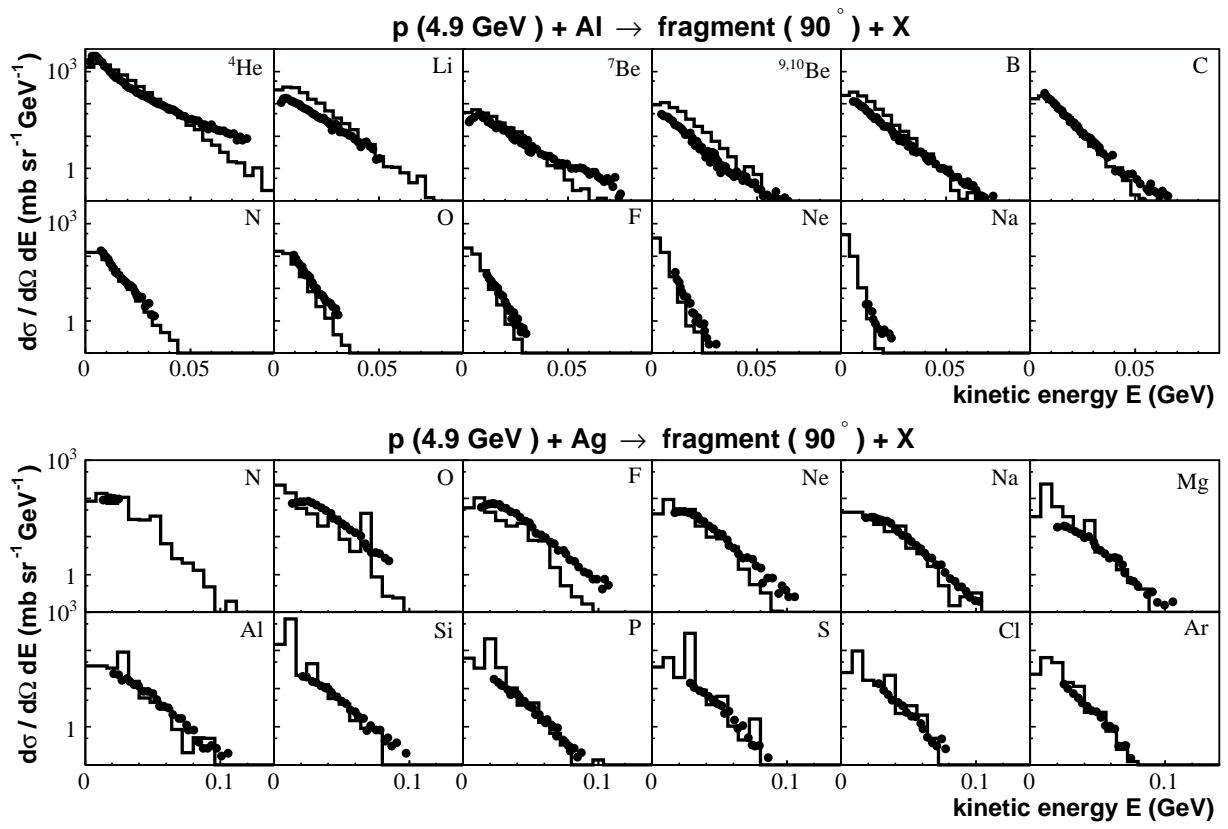

Figure 1. Energy spectra [14] of the nuclear fragments (symbols) indicated in the figure from $\mathrm{p}+\mathrm{Al}$ (upper panel) and $\mathrm{p}+\mathrm{Ag}$ (lower panel) reactions at $4.9 \mathrm{GeV}$ compared with ROC-model calculations (histograms) using $\Theta_{R}^{\max }=10 \mathrm{MeV}$ for $\mathrm{Al}$ and $\Theta_{R}^{\max }=6.8 \mathrm{MeV}$ for $\mathrm{Ag}$.

are taken as constant. A reasonable overall reproduction of the data could be achieved with $\Theta_{R}^{\max }$ taken as a function of the target mass number, which strongly decreases at light and becomes constant at heavy target masses. The fragment spectra result from a rather complicated superposition of contributions with different values of $\Theta_{R}(a, A)$, which are for small numbers of participants much smaller than $\Theta_{R}^{\max }$. The higher values of $\Theta_{R}^{\max }$ at low residue mass numbers do not necessarily imply a higher 'effective' $\Theta_{R}$ contributing to the spectra, because for light nuclei the partial cross sections drop more rapidly with increasing number of participants than in case of heavier nuclei. Anyway, decisive for the intended discussion of hadron production is the reproduction of existing data to get a realistic estimate of the energy consumed by fragmentation.

As example, figure 10 demonstrates that the basic features of fragment spectra are reasonably reproduced. In [2], we used $\Theta_{R}^{\max }=12 \mathrm{MeV}$ for light nuclei up to C, here $\Theta_{R}^{\max }=10 \mathrm{MeV}$ is taken for $\mathrm{Al}$ and $\Theta_{R}^{\max }=6.8 \mathrm{MeV}$ for $\mathrm{Ag}$ (see figure 1). For mass numbers above 100 the temperature is assumed to be constant and we take $\Theta_{R}^{\max }=6.8 \mathrm{MeV}$ also for $\mathrm{Pb}$. Since in the region around $\mathrm{Cu}$ there are no data available we (nonlinearly $\ddagger$ ) interpolate and get $\Theta_{R}^{\max }=7.5 \mathrm{MeV}$ for $\mathrm{Cu}$. It should be stressed that for the following considerations all other model parameters are the same as used in $[1,2]$ for describing the experimental results of [3] for interactions with carbon.

In figure 2 the momentum spectra of $\pi^{ \pm}, \mathrm{K}^{ \pm}$and $\overline{\mathrm{p}}$ from $\mathrm{pCu}, \mathrm{dCu}$ and $\alpha \mathrm{Cu}$ reactions obtained at KEK [3] are displayed. This experiment was motivated by $\ddagger$ All values of $\Theta_{R}^{\max }$ used are approximately described by $\Theta_{R}^{\max } / \mathrm{MeV}=6.8[1+1.2 \exp (-0.036 A)]$ with A being the target mass number. 


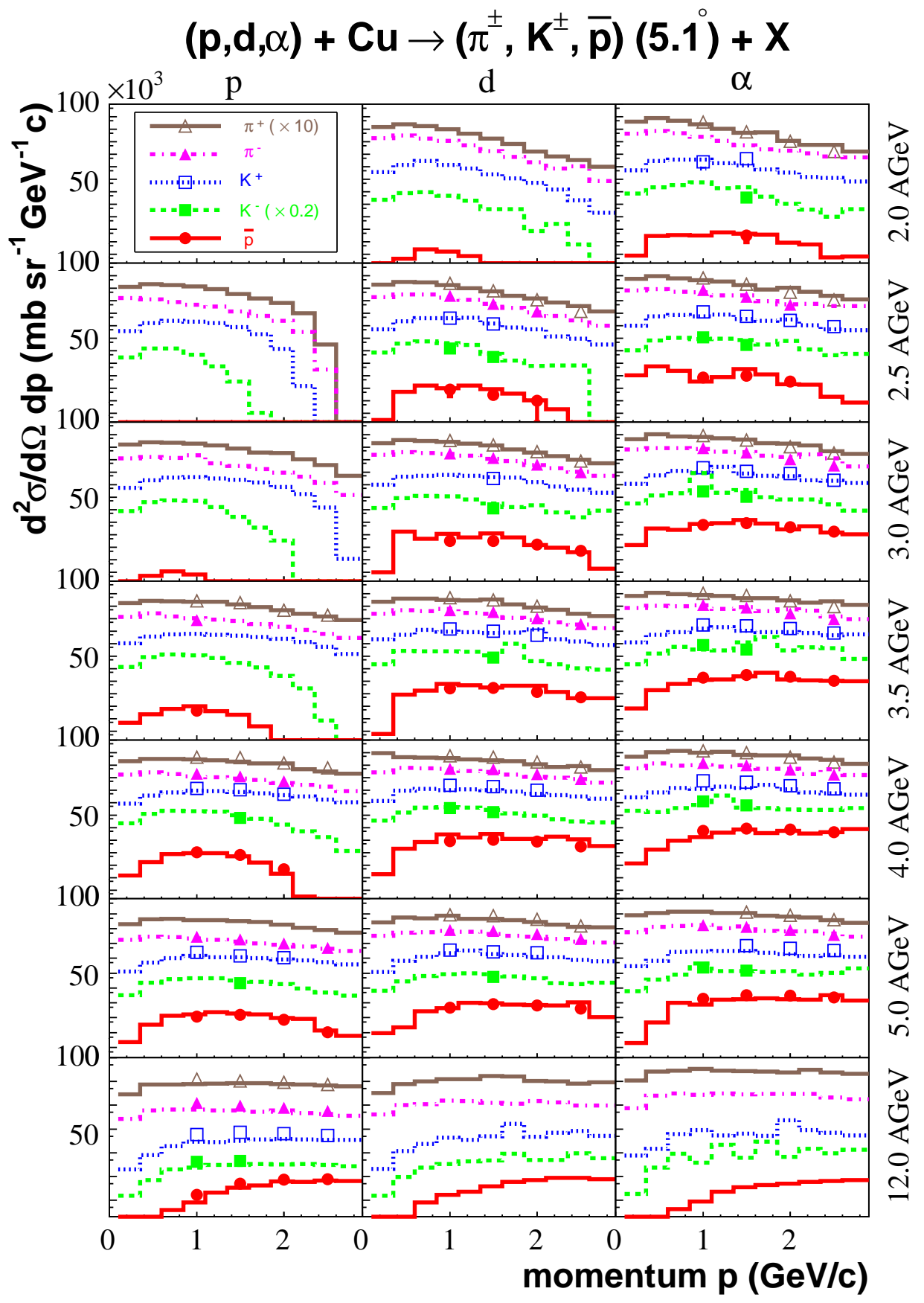

Figure 2. Momentum spectra of $\pi^{ \pm}, \mathrm{K}^{ \pm}$and $\overline{\mathrm{p}}[3]$ (symbols) from $\mathrm{pCu}$, $\mathrm{dCu}$ and $\alpha \mathrm{Cu}$ reactions compared with ROC-model calculations (histograms). Experimental and calculated results for $\pi^{+}$and $\mathrm{K}^{-}$mesons are multiplied by the factors indicated in the legend. The scale extends from $2 \times 10^{-8}$ to $10^{5} \mathrm{mb} \mathrm{sr}^{-1} \mathrm{GeV}^{-1} \mathrm{c}$ for energies up to $4.0 \mathrm{~A} \mathrm{GeV}$, while the lower limit is $2 \times 10^{-6}$ for $5.0 \mathrm{~A} \mathrm{GeV}$ and $2 \times 10^{-2} \mathrm{mb} \mathrm{sr}^{-1} \mathrm{GeV}^{-1} \mathrm{c}$ for $12.0 \mathrm{~A} \mathrm{GeV}$. 
measurements of large cross sections of subthreshold $\bar{p}$ production in nucleus-nucleus reactions at LBL-BEVALAC [20,21] and at GSI [22]. The authors [3] claimed that the use of light-ion beams for investigating subthreshold $\bar{p}$ production should be useful to verify theoretical models. Secondary effects like re-scattering, re-absorption, selfenergies, potentials etc, which hide and influence features of the primary production process, should be smaller than in heavy-ion reactions. Thus, it is the outstanding feature of the data that in $\mathrm{d}$ - and $\alpha$-induced reactions an enormous enhancement of $\overline{\mathrm{p}}$ production by nearly two and three orders of magnitude compared to p-induced interactions could be observed. This enhancement makes light-ion-induced reactions a candidate for the key to a deeper understanding of subthreshold $\bar{p}$ production in nucleus-nucleus reactions.

The KEK group [3] interprets their $\overline{\mathrm{p}}$ data by using the 'first-chance NN collision model' from [24] where the internal nucleon momenta were extracted from backward proton production [25] as a superposition of two Gaussian distributions. Momentum spectra and the incidence-energy dependence of p-induced reactions could be successfully reproduced by adapting one normalization parameter. However, the application of this model to d-induced reactions severely underestimates the $\bar{p}$ cross sections at subthreshold energies. Thus, according to [3], the effect cannot be explained by the internal motion of the nucleons in the deuteron.

In contrast, in $[26], \overline{\mathrm{p}}$ production in $\mathrm{p}+\mathrm{A}$ and $\mathrm{d}+\mathrm{A}$ reactions is analysed within a phase-space model incorporating the self-energies of the baryons. It is claimed that the internal momentum distribution of the deuteron provides a natural explanation of the large enhancement under discussion.

In [2] we reproduced the observed increase of subthreshold $\overline{\mathrm{p}}$ production in $\mathrm{dC}$ and $\alpha \mathrm{C}$ reactions compared to $\mathrm{pC}$ interactions and demonstrated that within the $\mathrm{ROC}$ model the number of participating nucleons is the key quantity for understanding subthreshold particle production. In addition, the whole set of experimental data for all ejectiles measured was well described. This is of special importance since there is not only the enhancement of $\bar{p}$ production, but also the increase of the $\mathrm{K}^{-}$cross sections with increasing energy and mass number of the projectile around the elementary production threshold at $2.6 \mathrm{GeV}$. Also the completely different energy and projectile mass dependence of the pion production cross sections far above the threshold could be reproduced.

Here, we repeat these considerations for the heavier target nuclei $\mathrm{Cu}$ and $\mathrm{Pb}$ and come to rather similar consequences. In figure 2] the results of the $\mathrm{ROC}$ model for $\mathrm{pCu}$, $\mathrm{dCu}$ and $\alpha \mathrm{Cu}$ reactions are compared to the data [3]. Again, the overall agreement is quite satisfactory in view of the different projectile types, the large region of incidence energies, the variety of ejectile species and the huge differences of many orders of magnitude in the considered cross section values. In case of the $\mathrm{Pb}$ target (see figure 3) only data for d-induced reactions up to $5 \mathrm{~A} \mathrm{MeV}$ and for p-induced reactions at $12 \mathrm{GeV}$ are available. Also for such a heavy target the reproduction of the data is satisfactory.

For $12 \mathrm{GeV}$ protons the ROC calculations tend to underestimate the $\mathrm{K}^{ \pm}$and $\overline{\mathrm{p}}$ data (see figures 2 and [3). In figure 4 a similar data set [23] is compared with ROC-model calculations. The spectra are measured at a slightly lower energy and at a similar angle but in a much wider momentum region compared to the KEK data. In this case the tendency of the ROC results goes in the opposite direction and overestimates the cross sections for the heavier ejectiles $\mathrm{K}^{ \pm}$and $\overline{\mathrm{p}}$. Also for the higher ejectile momenta the description of the whole data set for targets between 


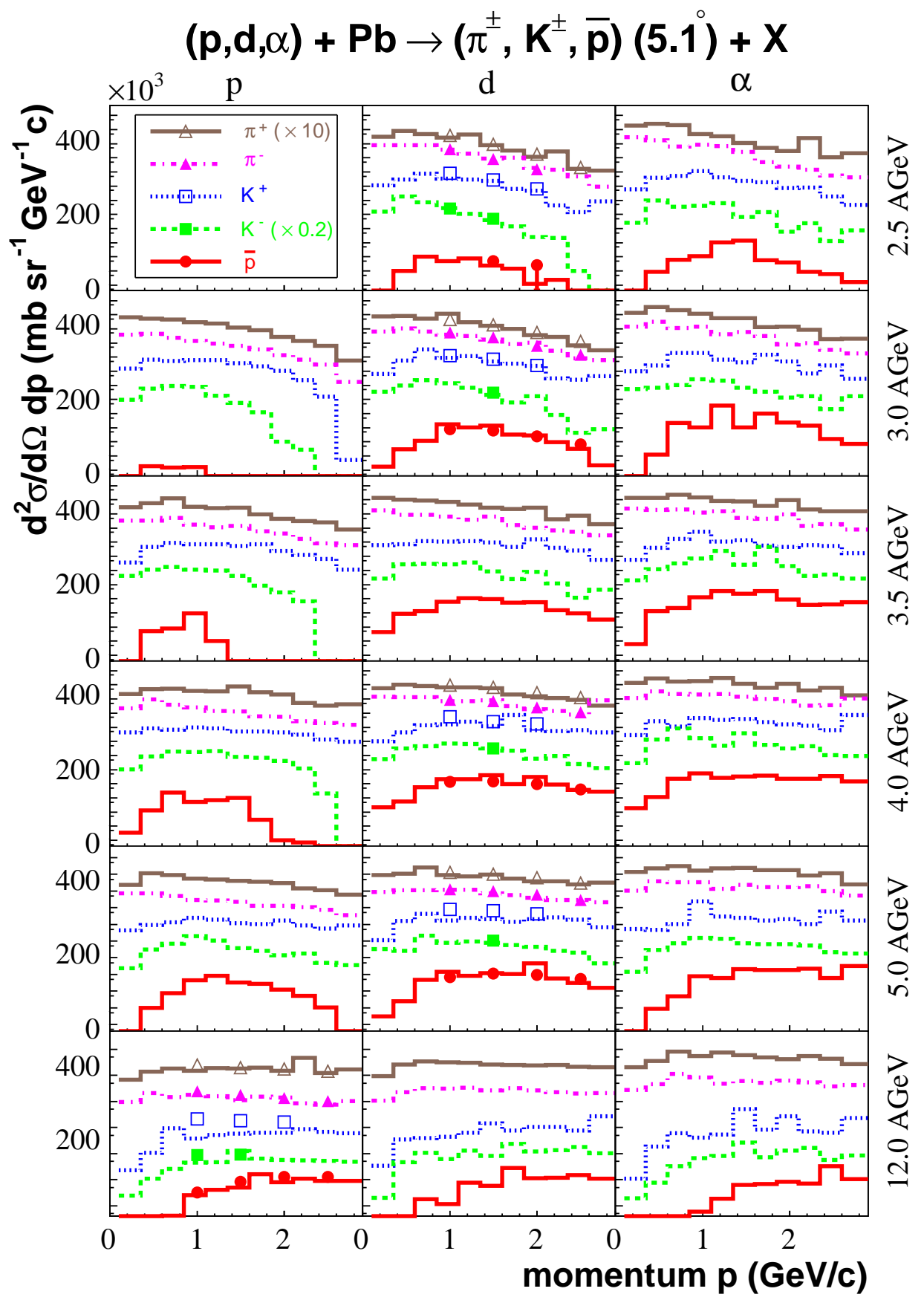

Figure 3. The same as figure 2 for $\mathrm{Pb}$ target. The scale extends from $4 \times 10^{-8}$ to $5 \times 10^{5} \mathrm{mb} \mathrm{sr}^{-1} \mathrm{GeV}^{-1} \mathrm{c}$ for energies up to $4.0 \mathrm{~A} \mathrm{GeV}$, while the lower limit is $4 \times 10^{-6}$ for $5.0 \mathrm{~A} \mathrm{GeV}$ and $4 \times 10^{-2} \mathrm{mb} \mathrm{sr}^{-1} \mathrm{GeV}^{-1} \mathrm{c}$ for $12.0 \mathrm{~A} \mathrm{GeV}$. 


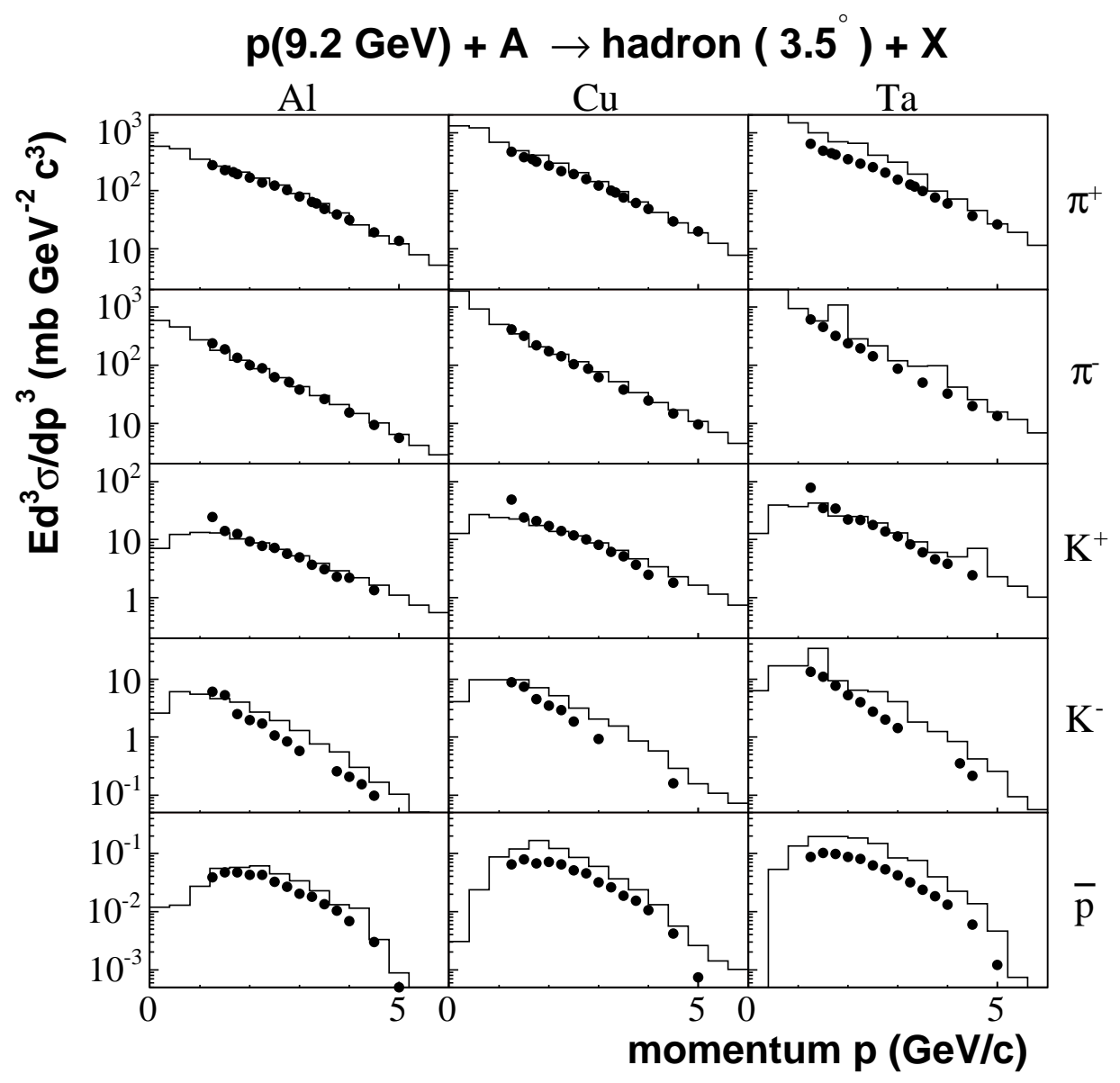

Figure 4. Momentum spectra of $\pi^{ \pm}, K^{ \pm}$and $\bar{p}$ [23] (symbols) compared to ROC-model calculations (histograms).

Table 1. Calculated cross sections and statistical errors $\sigma_{p}, \sigma_{d}$ and $\sigma_{\alpha}$ for $\bar{p}$ production in $\mathrm{p}-, \mathrm{d}$ - and $\alpha$-induced interactions, respectively, with $\mathrm{Cu}$ at 3.5 A GeV for different temperature parameters $\Theta_{R}^{\max }$.

\begin{tabular}{cccc}
\hline$\Theta_{R}^{\max }(\mathrm{MeV})$ & $\sigma_{p}(\mathrm{nb})$ & $\sigma_{d}(\mu \mathrm{b})$ & $\sigma_{\alpha}(\mu \mathrm{b})$ \\
\hline 7.0 & $5.2 \pm 0.2$ & $0.57 \pm 0.03$ & $1.7 \pm 0.1$ \\
7.5 & $2.7 \pm 0.1$ & $0.39 \pm 0.05$ & $1.5 \pm 0.2$ \\
8.0 & $1.9 \pm 0.2$ & $0.27 \pm 0.02$ & $1.2 \pm 0.2$ \\
\hline
\end{tabular}

Be (see figure 3 in [1]) and Ta is satisfactory. Typically, the deviations between our calculations and the data are less than a factor of 2 .

Also an assumed uncertainty of $0.5 \mathrm{MeV}$ for the temperature parameter $\Theta_{R}^{\max }$ yields deviations within this limit at the lowest energy where the enhancement effect was observed (see table 1). As shown in [1] the dependence of the calculated results on $\Theta_{R}^{\max }$ becomes smaller with increasing projectile energy. From table 1 can be deduced 
that at a given incidence energy per nucleon an increasing projectile mass diminishes the dependence of the subthreshold production cross section on the temperature parameter $\Theta_{R}^{\max }$ of the residual nucleus, too. This can be explained by the larger effective number of participants in case of the heavier projectile, which leads to an increase of the energy available for particle production.

A striking feature of the obtained results is the consistent quality of data reproduction for all types of final particles independent of the target mass. With regard to the fact that the cross sections for the interaction of the various final particles with nucleons differ considerably one should, especially for heavy target nuclei, expect distinct deviations of the calculated spectra from the measured ones, since individual properties of the various outgoing particles like the mentioned cross sections are not (yet) incorporated in the model. In the light of this result the question of the treatment of final-state interactions is worth being discussed. It is the excitation of the residual nucleus which diminishes the energy available for particle production in the model calculations. This decreases the corresponding cross sections especially in the threshold region independent of the type of secondary particles. It seems that individual interactions of the outgoing particles with the target nucleons do not play a decisive role. A rigorous explanation would be the assumption of a finite life time of intermediate states called FBs in the ROC model. If such a life time would be comparable to the time the FBs need to leave the residual nucleus, then the individual properties of the various particles would wash-out. We are aware of the difficulty to articulate such a point of view regarding the fact that a major topic of present research activities consists in investigating medium modifications of individual particle properties. On the other hand, although the deviations between our calculations and the data are usually less than a factor of 2 or so, there remains room for not too large effects not yet included in the ROC model.

As shown in [2] and supported by the present results, the main contribution for subthreshold particle production in conditions of the experiments [3] comes, according to the ROC model, from the excitation of FBs with baryon numbers from 2 to 4 . This means that physical properties of such FBs may be directly studied in collisions of proton, deuteron and helium-ion beams with the lightest nuclear targets, ${ }^{2} \mathrm{H},{ }^{3} \mathrm{He}$ and ${ }^{4} \mathrm{He}$. In this case such complicating effects like varying numbers of participating nucleons, loss of energy for the excitation of the residual nucleus, final-state absorption and rescattering in the residue, are significantly diminished or removed at all. The model reveals a complete analogy of the interaction of few-nucleon groups to the interaction of single nucleons in the subthreshold production processes. This may be treated as an indication that properties of few-nucleon groups in conditions of high energy-momentum transfer are governed by quark-gluon degrees of freedom. That makes experiments on subthreshold production at light target nuclei a promising tool for the study of the transition from hadronic to quark-gluon properties.

\section{Summary}

Subthreshold particle production is a collective phenomenon which is far from being completely understood. From the viewpoint of the ROC model, data on subthreshold particle production can be reproduced by considering the interaction of few-nucleon groups in complete analogy to the interaction of single nucleons, also with regard to high-momentum transfer processes. It has been demonstrated here that in a wide range of target mass numbers the cluster concept yields a quite natural explanation 
of the enhancement of subthreshold particle production due to the energy gain in the interaction of few-nucleon groups compared to NN interactions. Especially at energies below or near the NN threshold the consideration of the interplay between hadron production and fragmentation processes is important for the reproduction of the cross sections. This concept should be applicable not only in proton- or light-ion-induced reactions, but also for heavy-ion interactions, although in the latter case the number of partial processes increases tremendously. In this sense, the ROC model can be considered as a promising approach to a unified description of particle production processes in a large variety of different types of nuclear reactions.

\section{Acknowledgments}

One of the authors (H.M.) would like to thank W. Enghardt for the promotion of this study.

\section{References}

[1] Komarov V I, Müller H and Sibirtsev A 2004 J. $\quad$ Phys. $\quad$ G. $30 \quad 921$. http://arXiv.org/nucl-th/0312087

[2] Müller H and Komarov V I 2004 J. Phys. G. 30 1379. http://arXiv.org/nucl-th/0402009

[3] Sugaya Y et al. 1998 Nucl. Phys. A634 115

[4] Glauber R J and Mathiae J 1970 Nucl. Phys. B 21135

[5] Knoll J 1979 Phys. Rev. C 20773

[6] Knoll J 1980 Nucl. Phys. A 343511

[7] Bohrmann S and Knoll J 1981 Nucl. Phys. A 356498

[8] Shyam R and Knoll J 1984 Nucl. Phys. A 426606

[9] Shyam R and Knoll J 1986 Nucl. Phys. A 448322

[10] Knoll J and Shyam R 1988 Nucl. Phys. A 483711

[11] Ghosh B and Shyam R 1990 Phys. Lett. B 234248

[12] Ghosh B 1992 Phys. Rev. C 45 R518

[13] Müller H 2001 Eur. Phys. J. C 18 563. http://arXiv.org/hep-ph/0011350

[14] Westfall G et al. 1978 Phys. Rev. C17 1368

[15] Müller H and Sistemich K 1992 Z. Phys. A 344197

[16] Katkoff S, Fickel H R and Wyttenbach A 1968 Phys. Rev. 1661147

[17] English G and Porile N 1974 Phys. Rev. C 102268

[18] Kaufman S, Weisfield M, Steinberg E, Wilkins B and Henderson D 1976 Phys. Rev. C14 1121

[19] Kaufman S and Steinberg E 1980 Phys. Rev. C22 167

[20] Caroll J et al. 1989 Phys. Rev. Lett. 621829

[21] Shor A et al. 1989 Phys. Rev. Lett. 632192

[22] Schröter A et al. 1993 Nucl. Phys. A $\mathbf{5 5 3} 775 \mathrm{c}$

[23] Vorontsov I A, Safronov G A, Sibirtsev A A, Smirnov G N and Trebukhovsky Y V 1988 ITEP$11-1988$

[24] Shor A, Perez-Mendez V and Ganezer K 1990 Nucl. Phys. A 514717

[25] Geaga J V et al. 1980 Phys. Rev. Lett. 451993

[26] Cassing W, Lykasov G and Teis S 1994 Z. Phys. A 348247 